\title{
Microrheology and Release Behaviors of Self-Assembled Steroid Hydrogels
}

\author{
W. Liang1, J. R. Guman-Sepulveda'2, S. He1, A. Dogariu'2, J. Y. Fang1 ${ }^{*}$ \\ ${ }^{1}$ Department of Materials Science and Engineering, The College of Optics and Photonics, University of Central \\ Florida, Orlando, USA \\ ${ }^{2}$ CREOL, The College of Optics and Photonics, University of Central Florida, Orlando, USA \\ Email: ${ }^{*}$ Jiyu.fang@ucf.edu
}

Received 28 June 2015; accepted 26 July 2015; published 29 July 2015

Copyright (C) 2015 by authors and Scientific Research Publishing Inc.

This work is licensed under the Creative Commons Attribution International License (CC BY).

http://creativecommons.org/licenses/by/4.0/

(c) (i) Open Access

\section{Abstract}

A hydrogel is formed by the self-assembly of sodium deoxycholate (NaDC) in aqueous solution with sodium chloride at pH-7.0. The NaDC hydrogel made of the three-dimensional network of nanofibers shows pH-dependent swelling behaviors. Polystyrene particles with a diameter of 100 $\mathrm{nm}$ and doxorubicin hydrochloride (DOX) can be easily loaded into the NaDC hydrogel through swelling. By using the loaded polystyrene particles as a light scattering probe, we study the microrheology of the NaDC hydrogel, showing complex viscoelastic properties. The viscous component dominates at both low and high frequencies, while the elastic component dominates in the intermediate range. The cavity size of the nanofiber network can also be estimated to be $\sim 180 \mathrm{~nm}$. We show that the loaded DOX can be slowly released from the hydrogels into aqueous solution. The release profile of DOX is found to depend on the $\mathrm{pH}$ value of the solution.

\section{Keywords}

Sodium Deoxycholate, Self-Assembly, Hydrogels, Microrheology, Swelling, Release

\section{Introduction}

Supramolecular hydrogels have shown potential applications in drug delivery, wound healing, and tissue engineering [1]-[5]. They can be simply formed by the self-assembly of small molecules in aqueous solution through noncovalent interactions including hydrogen bonding, $\pi-\pi$ stacking, electrostatic, and van der Waals interactions.

Bile salts are a biological surfactant which is synthesized in the liver for aiding the digestion of lipids in the small intestine [6] [7]. Unlike traditional surfactants, which typically have a hydrophilic head group bonded to a

\footnotetext{
${ }^{*}$ Corresponding author.
}

How to cite this paper: Liang, W., Guman-Sepulveda, J.R., He, S., Dogariu, A. and Fang, J.Y. (2015) Microrheology and Release Behaviors of Self-Assembled Steroid Hydrogels. Journal of Materials Science and Chemical Engineering, 3, 6-15. 
linear, flexible, and hydrocarbon tail, bile acids have a rigid and quasi-planar steroid backbone with polar hydroxyl groups on the hydrophilic $\alpha$ face and methyl groups on the hydrophobic $\beta$ face, and a carboxyl group linked to the steroid backbone through a short alkyl chain. The facial amphiphilic structure of bile salts makes them a unique building block for the assembly of one-dimensional (1D) supramolecular structures such as nanofibers, nanoribbons, and nanotubes [8]-[22]. Under certain conditions, these 1D supramolecular structures are able to further entangle into three-dimensional (3D) networks, leading to the formation of hydrogels.

The formation of hydrogels from the self-assembly of bile salts in aqueous solution have been known for a long time [23] [24]. For example, sodium lithocholate (monohydroxy bile salt) is able to form hydrogels at $\mathrm{pH}-$ 12.0 [18], and sodium deoxycholate ( $\mathrm{NaDC}$ ) (dihydroxy bile salt) forms transparent hydrogels at $\mathrm{pH}-7.0$ [23]. However, sodium cholate (trihydroxy bile salt) is not able to form hydrogels. The transparent NaDC hydrogels formed at the neutral $\mathrm{pH}$ are particularly interesting. X-ray diffraction studies showed the formation of supramolecular helical structures during the gelling process of NaDC [24]. Steady-state and lifetime fluorescent measurements by using pyrene as a probe suggested that NaDC hydrogels consisted of entangled polymer-like aggregates [25].

In this paper, we report the pH-dependent swelling behavior of NaDC hydrogels. By using the polystyrene particles loaded in NaDC hydrogels through swelling as a light scattering probe, we study the microrheology of NaDC hydrogels, revealing complex viscoelastic properties. The doxorubicin hydrochloride (DOX) loaded in NaDC hydrogels can be slowly released into aqueous solution. The release profile of DOX varies as a function of $\mathrm{pH}$ values.

\section{Experimental Section}

\subsection{Materials}

Sodium chloride $(\mathrm{NaCl})$, hydrogen chloride $(\mathrm{HCl})$, doxorubicin hydrochloride (DOX), and sodium deoxycholate (NaDC) were purchased from Sigma-Aldrich. NaDC and DOX were used as received without further purification. Polystyrene particles with the diameter of $\sim 100 \mathrm{~nm}$ were obtained from Thermo Scientific. Water used in our experiments was purified with Easypure II system (18 M $2 / \mathrm{cm}, \mathrm{pH} 5.7)$.

\subsection{Hydrogel Formation}

Hydrogel formation: For the formation of hydrogels, $82.91 \mathrm{mg} \mathrm{NaDC}$ was added into $2 \mathrm{~mL}$ aqueous solution containing $0.02 \mathrm{M} \mathrm{NaCl}$ at $\mathrm{pH}$ 8.9. $\mathrm{NaDC}$ solution was vortexed for $5 \mathrm{~min}$ at $\sim 60^{\circ} \mathrm{C}$ and then cooled to room temperature. The hydrogel formed when the $\mathrm{pH}$ of $\mathrm{NaDC}$ solution was adjusted to 7.0.

\subsection{Hydrogel Characterization}

Hydrogel characterization: NaDC hydrogels were dried on glass substrates and characterized with an atomic force microscope (Dimension 3100 AFM from Veeco Instruments) in air at room temperature. A silicon nitride cantilever (Nanosensors) with a spring constant of $30 \mathrm{~N} / \mathrm{m}$ and a resonant frequency of $260 \mathrm{kHz}$ was used in tapping mode. The size of the cantilever tips (radius of curvature) is about $15 \mathrm{~nm}$ according to the manufacturer. The swelling behavior of $\mathrm{NaDC}$ hydrogels in a glass vial was studied by measuring their height increases in over time. In our experiments, $4 \mathrm{~mL}$ of $0.172 \mathrm{mM}$ DOX aqueous solution with varied $\mathrm{pH}$ values was added on the top of 2 $\mathrm{mL} \mathrm{NaDC}$ hydrogels. Polystyrene particles were added in aqueous solution at a ratio of $10 \% \mathrm{w} / \mathrm{w}$ at $\mathrm{pH} 7.0$. The solution was then added to the top of NaDC hydrogels in a glass vial at room temperature. The amount of loaded polystyrene particles through the swelling was estimated to $0.05 \mathrm{~g}$ in $1.0 \mathrm{~g}$ hydrogels. An optical fiber based setup using a low-coherence light source was utilized to detect the dynamic light scattering from the polystyrene particles loaded in NaDC hydrogels. The local viscoelastic properties of the hydrogels were determined by the frequency analysis of the intensity fluctuations of the scattered light.

\subsection{DOX Release from Hydrogels}

DOX release from hydrogels: After the swelling of NaDC hydrogel reaches the equilibrium, excess DOX solution was removed from the top of the swelled hydrogel with a pipette. $3 \mathrm{~mL}$ of aqueous solution with different $\mathrm{pH}$ values was then added on the top of the DOX-loaded hydrogel formed by the swelling at the corresponding $\mathrm{pH}$ 
values in a glass vial. The aqueous solution was then removed with a pipette each hour. The concentration of DOX released in the removed aqueous solution was characterized with a Cary $100 \mathrm{UV}$-vis spectrophotometer.

\section{Results and Discussion}

The chemical structure of sodium deoxycholate $(\mathrm{NaDC})$ is shown in Figure 1. It has two polar hydroxyl groups on the $\alpha$ face and three methyl groups on the $\beta$ face of the steroid backbone. In our experiments, $\mathrm{NaDC}$ was added into aqueous solution containing $\mathrm{NaCl}$ at $\mathrm{pH}$ 8.9. The gelling of NaDC solution occurs when the $\mathrm{pH}$ of the solution is reduced to 7.0 (Figure 1), which is in agreement with the result reported in the literature [23] [24]. Atomic force microscopy (AFM) measurements show that NaDC hydrogels are made of the network of worm-like nanofibers and nanofiber bundles (Figure 1). The diameter of the nanofibers measured from the height of the nanofibers shown in the AFM image is $65 \pm 20 \mathrm{~nm}$. The $\mathrm{pK}_{\mathrm{a}}$ value of deoxycholate molecules is 6.6 [23]. Thus, some of the deoxycholate molecules are expected to be protonated at $\mathrm{pH}$ 7.0. The gelling at $\mathrm{pH} 7.0$ suggests that the hydrogen bonding of deoxycholate molecules may play an important role for the formation of NaDC hydrogels.

$\mathrm{NaDC}$ hydrogels show $\mathrm{pH}$-dependent swelling behaviors. In our experiments, $4 \mathrm{~mL}$ of DOX aqueous solution with varied $\mathrm{pH}$ values on the top of $2 \mathrm{~mL} \mathrm{NaDC}$ hydrogels in a glass vial at room temperature (Figure 2). The swelling of the hydrogels is clearly evident from the increase of their heights in the glass vial over time. After the swelling of the hydrogels reaches a saturated condition, excess DOX solution could be removed from the top of the swelled hydrogels with a pipette (Figure 2). The swelled hydrogels remain stable in an upturned glass and show fluorescence when being excited with UV light (Figure 2), suggesting that DOX is loaded into the hydrogels through swelling. The swelling ratio of NaDC hydrogels is defined as $\left(\mathrm{H}_{\mathrm{t}}-\mathrm{H}_{0}\right) / \mathrm{H}_{0}$, where $\mathrm{H}_{0}$ is the height of the hydrogel in the glass vial before the swelling of DOX solution and $\mathrm{H}_{\mathrm{t}}$ is the height of the swelling hydrogel at time $\mathrm{t}$. As can be seen from Figure 2, the swelling ratio of NaDC hydrogels slowly increases over time and reach the saturated value of $\sim 110$ after 250 hours (defined as the "equilibrium" swelling ratio). The equilibrium swelling ratio reaches $\sim 140$ at $\mathrm{pH} 10.0$. However, it drops to $\sim 90$ when the $\mathrm{pH}$ value is increased to 11.0 (Figure 2). A further increase of $\mathrm{pH}$ values causes the dissocia tion of NaDC hydrogels. The balance between the hydrogen bonding and the electrostatic repulsion of nanofiber networks determines the swelling behavior of NaDC hydrogels. As the $\mathrm{pH}$ value increases, the degree of the ionization of deoxycholate molecules increases, which would enlarge the cavity of the nanofiber networks due to the increased electrostatic repulsion of the nanofibers and consequently cause the increase of the swelling ratio of NaDC hydrogels. On the other hand, the hydrogel bonding would be cleaved when all
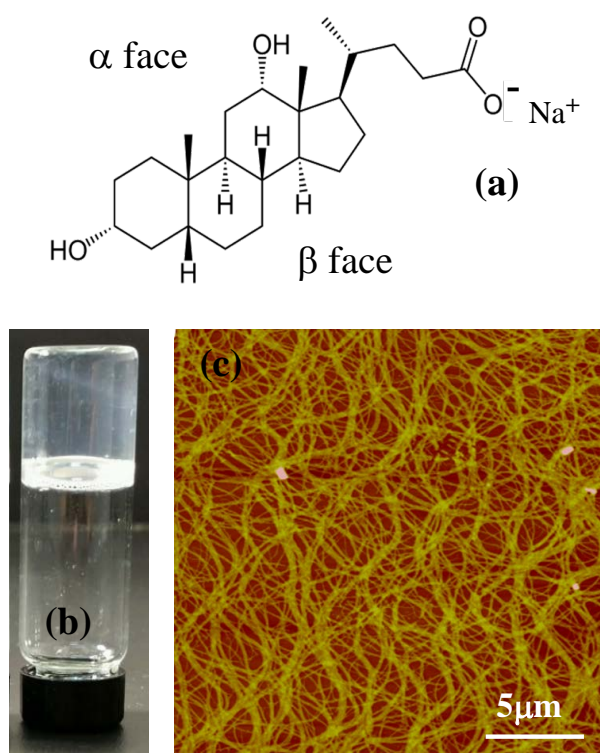

Figure 1. (a) Chemical structure of NaDC; (b) Photography of NaDC hydrogels formed at pH-7.0 in an upturned glass vial; (c) AFM image of NaDC hydrogels dried on a glass substrate. 

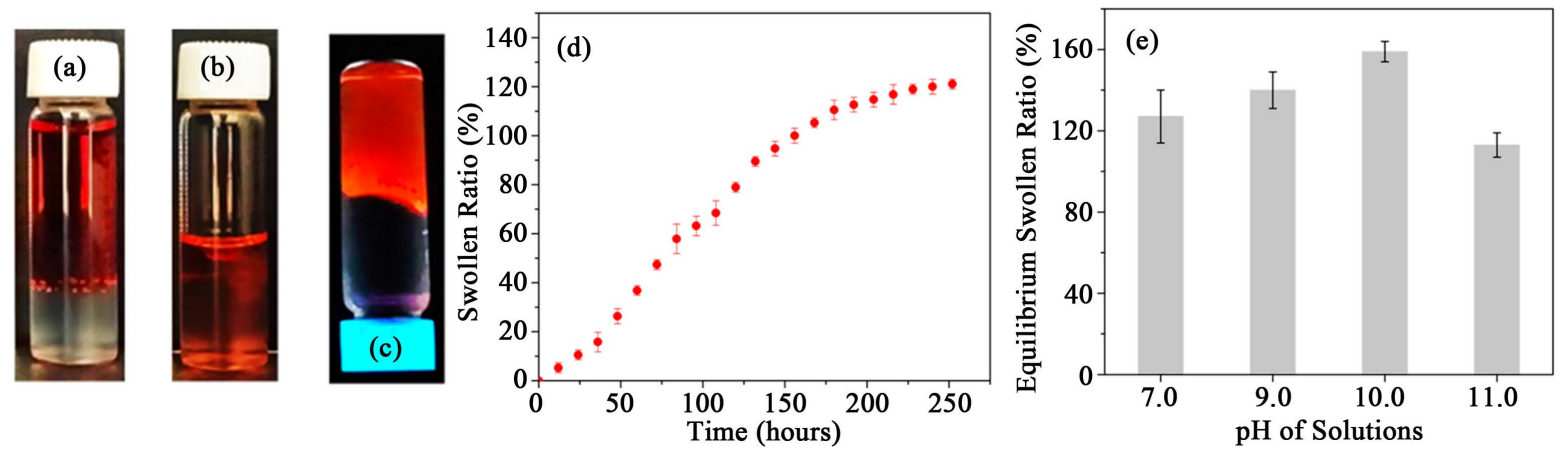

Figure 2. (a) Photograph of NaDC hydrogels in a glass vial after the addition of DOX solution at pH 7.0; (b) Photograph of DOX-loaded NaDC hydrogels through swelling at pH 7.0; (c) Emission of DOX-loaded NaDC hydro-gels in an upturned glass vial under UV illumination; (d) Swelling kinet-ics of NaDC hydrogels at pH 7.0 at room temperature; (e) Equilibrium swelling ratio of $\mathrm{NaDC}$ hydrogels at different $\mathrm{pH}$ values.

the deoxycholate molecules are ionized at high $\mathrm{pH}$ values, which causes the dissociation of NaDC hydrogels.

The DOX loaded in NaDC hydrogels through swelling can be released into aqueous solution. In our experiments, $3 \mathrm{~mL}$ of aqueous solution with varied $\mathrm{pH}$ values was added on the top of NaDC hydrogels, in which DOX was loaded by swelling at the corresponding $\mathrm{pH}$ values. We note that the loaded DOX is slowly released from the hydrogels into the upper aqueous solution. Periodically, the upper aqueous solution was removed from the top of the hydrogels. After being characterized with UV-vis spectroscopy, the removed solution was added back to the top of the hydrogel. Figure 3 shows the UV-Vis spectra of DOX in the removed aqueous solution as a function of time at $\mathrm{pH}$ 7.0. The absorbance of DOX at $\sim 500 \mathrm{~nm}$ increases over time, which suggests the continuous release of DOX from the hydrogel to the upper aqueous solution. Thus, we used the absorbance at $\sim 500 \mathrm{~nm}$ to quantify the cumulative percent release of DOX from the hydrogel as a function of time at different $\mathrm{pH}$ values (Figure 3). The release rate increases as the increase of $\mathrm{pH}$ values. At $\mathrm{pH} 11, \sim 80 \%$ of the initially loaded DOX is released into the upper aqueous solution after 10 hours. However, only $25 \%$ of the initially loaded DOX is released into the upper solution after 10 hours at $\mathrm{pH} 7.0$. We analyzed the release profiles with Ritger-Peppas equation [26]:

$$
M_{t} / M_{\infty}=k t^{n}
$$

where $M_{t} / M_{\infty}$ is the fractional solute release, $t$ is the release time, $k$ is a constant, and $n$ is the diffusional exponent characteristic of the release mechanism. If Fickian diffusion dominates, $n$ value should be less than 0.5. However, we find that the fitted $n$ value is 0.56 at $\mathrm{pH} 7.0,0.63$ at $\mathrm{pH} 9.0,0.72$ at $\mathrm{pH} 10.0$, and 0.80 at $\mathrm{pH}$ 11.0, respectively (Figure 3). This result indicates that the release of the DOX from NaDC hydrogels is mainly controlled by hydrogel relaxations.

The swelling behavior of NaDC hydrogels also allows us to load polystyrene particles into the hydrogels. In our experiments, the amount of loaded polystyrene particles was estimated to be $0.05 \mathrm{~g}$ in $1.0 \mathrm{~g} \mathrm{NaDC}$ hydrogels at $\mathrm{pH} 7.0$, which gives a mass concentration of polystyrene particles less than $0.5 \%$. We used the loaded polystyrene particles as a light scattering probe to passively measure the local viscoelastic behavior of NaDC hydrogels. The local viscoelasticity of the hydrogels influences the Brownian motion of the loaded particle probe, which can be characterized by the temporal evolution of their mean square displacement (MSD), $\left\langle\Delta \mathrm{r}^{2}(\mathrm{t})\right\rangle$ [27] [28]. The fluctuation of the scattered light due to the random displacement of the loaded particle probe can be analyzed in the frequency domain by using the fiber-based, low-coherence dynamic light scattering technique (LC-DLS) [29]. This technique allows to isolate the single-scattering component even in optically dense media. It has been shown that the associated power spectrum of the intensity fluctuations can be represented as a multi-Lorentzian form [30] [31].

$$
P(f)=\frac{2}{\pi} \sum_{i=1}^{M} \frac{a_{i} / \tau_{i}}{f^{2}+\left(1 / \tau_{i}\right)^{2}}, \quad \text { subject to } \sum_{i=1}^{M} a_{i}=1
$$

Figure 4 shows typical experimental power spectra of the scattering from the NaDC hydrogels with and without polystyrene particles. The power spectra ranging from $5 \mathrm{~Hz}$ to $25 \mathrm{kHz}$ were recorded over a period 


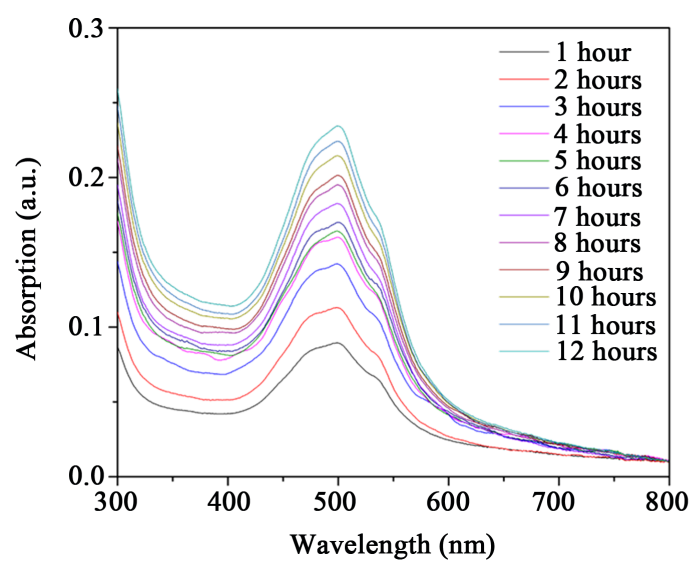

(a)

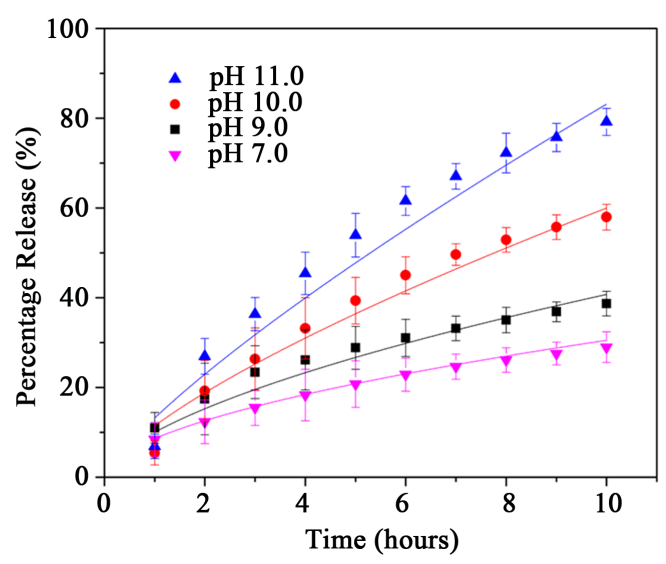

(b)

Figure 3. (a) UV-vis spectra of time-dependent release of DOX from NaDC hydrogels to the upper aqueous solution at $\mathrm{pH} 7.0$; (b) Cumulative release profiles of DOX from NaDC hydrogels to the upper aqueous solution at different $\mathrm{pH}$ values. The solid curves were the fitted results with Ritger-Peppas equation.

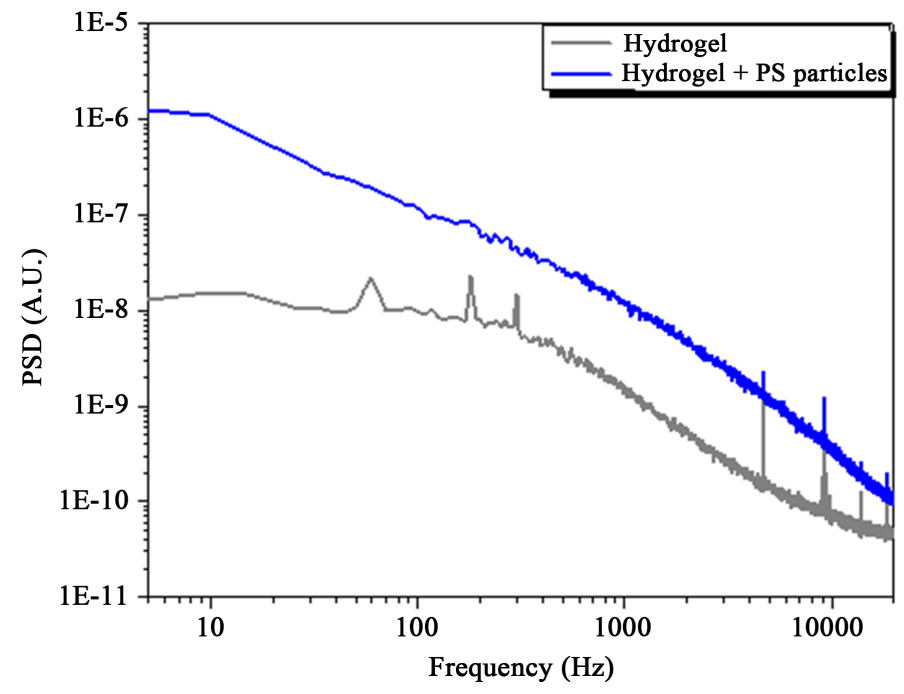

Figure 4. Experimental power spectra of scattered intensity fluctuations of NaDC hydrogels with and without polystyrene (PS) particle probes.

of $10 \mathrm{~s}$ of continuous sampling and then a total of 60 samples were averaged during a total acquisition time of $10 \mathrm{~min}$. As can be seen from Figure 4, a significantly stronger scattering is obtained from the hydrogel with polystyrene particles. Thus, we further analyze the scattering from the hydrogel with loaded particle probes.

According to the single-scattering model for spherical particles, the Fourier Transform of the power spectrum of the fluctuations relates to MSD [32]:

$$
F\{P(f)\}=\exp \left[-\frac{q^{2}}{6}\left\langle\Delta r^{2}(t)\right\rangle\right]
$$

Since the power spectrum has a Lorentzian shape, its Fourier Transform can be directly expressed as the superposition of multiple decaying exponentials:

$$
F\{P(f)\}=F\left\{\frac{2}{\pi} \sum_{i=1}^{N} \frac{a_{i} / \tau_{i}}{f^{2}+\left(1 / \tau_{i}\right)^{2}}\right\}=\sum_{i=1}^{N} a_{i} \exp \left(-2 \pi t / \tau_{i}\right)
$$


This allows us to calculate the time evolution of the MSD in terms of numerical parameters obtained from fitting the normalized power spectrum of the intensity fluctuations to the multi-Lorentzian model:

$$
\left\langle\Delta r^{2}(t)\right\rangle=-\frac{6}{q^{2}} \ln \left[\sum_{i=1}^{N} a_{i} \exp \left(-2 \pi t / \tau_{i}\right)\right], q=\frac{4 \pi n \sin (\theta / 2)}{\lambda_{0}}
$$

where $q$ is the magnitude of the scattering vector, $n$ is the index of refraction of the medium (i.e. DI water), $\lambda_{0}$ is the central wavelength of the source (680 nm in our experiments), and $\theta$ is the scattering angle $(\theta=\pi$ for the backscattering configuration used here). $\mathrm{q}$ is calculated to be $2.46 \times 10^{7} \mathrm{~m}^{-1}$.

The calculated MSD of the particle probes is presented in Figure 5. The MSD clearly exhibits three different regimes. At short times, the MSD is governed by the normal diffusion of the particles in the aqueous medium and therefore grows linearly with time. At intermediate times the MSD increase slows down considerably while at longer times the normal linear increase is reached again. This is a typical behavior of particle probes in worm-like micelle solution [32] [34].

A quantitative description can be pursued in terms of the particle probes confined in the cavities of worm-like micelle networks. Following the model outlined in Refs [34] [35]. The MSD corrected for multi-exponential relaxation times evolves in time as:

$$
\left\langle\Delta r^{2}(t)\right\rangle=6 \delta^{2}\left(1-\mathrm{e}^{\left.-\left(\frac{D_{0}}{\delta^{2}}\right)^{a}\right)^{1 / a}}\left(1+\frac{D_{m}}{\delta^{2} t}\right)\right.
$$

where $6 \delta^{2}$ represents an average cavity size, $\mathrm{D}_{0}$ is the diffusion coefficient of the particles in the aqueous medium considered to be a Maxwellian fluid, $\mathrm{D}_{\mathrm{m}}$ is the diffusion in the worm-like micelle system, and $a$ is a constant value correcting for multiple relaxation times ( $a=1$ in the case of monoexponential relaxation).

In our experiments, $\mathrm{D}_{0}$ was evaluated as the diffusion coefficient of free particles in water while the parameters $\delta, \mathrm{D}_{\mathrm{m}}$, and $a$ were considered to be the fitting parameters for the evaluated $\left\langle\Delta r^{2}(t)\right\rangle$. As can be seen in Figure 5, Equation (5) fits well to the experimental data $\left(\mathrm{R}^{2}=0.9998\right)$, giving $a=0.605, D_{m}=0.369$ $\mu \mathrm{m}^{2} / \mathrm{s}$, and $\delta=0.0394 \mu \mathrm{m}$. The average cavity size of the nanofiber network of NaDC hydrogels is estimated to be $\sim 180 \mathrm{~nm}$.

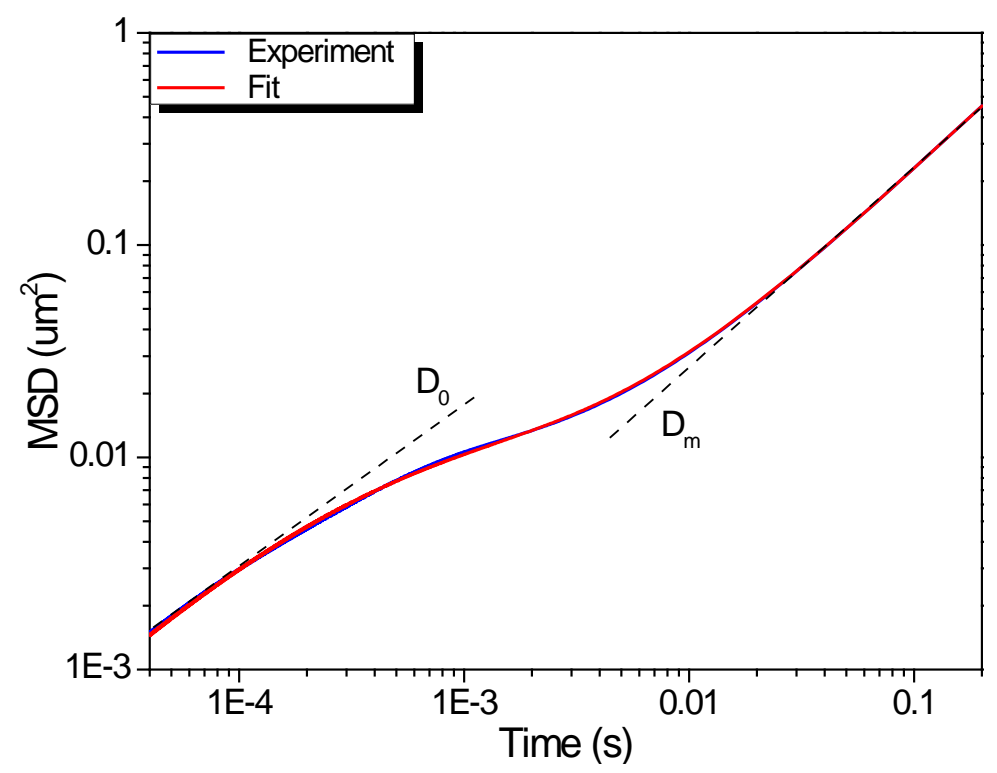

Figure 5. Temporal evolution of the mean square displacement calculated from the power spectra of scattered intensity fluctuations from PS particle-loaded hydrogels, together with the fitted curve with Equation (5), in which $a=0.605, D_{m}$ $=0.369 \mu \mathrm{m}^{2} / \mathrm{s}$, and $\delta=0.0394 \mu \mathrm{m} . D_{0}$ and $D_{m}$ are diffusion constants in the short-and long-time regimes, respectively. 
The temporal evolution of $\left\langle\Delta r^{2}(t)\right\rangle$ can be further related to the viscoelastic modulus, $G^{*}(f)$, by solving the general Langevin equation of motion with a memory function which is incorporated to take into account the viscoelastic properties of the fluid [33]-[35]. This can be approached by using the log-derivative method [36] where the power law describing the MSD

$$
\left|G^{*}(f)\right| \approx \frac{k_{B} T}{\pi a\left\langle\Delta r^{2}(1 / f)\right\rangle \Gamma[1+\alpha(f)]}
$$

is bounded between 0 (elastic confinement) and 1 (viscous diffusion). The magnitude and components of the viscoelastic modulus can be then calculated to be

$$
\begin{aligned}
& G^{\prime}(f)=\left|G^{*}(f)\right| \cos (\pi \alpha(f) / 2) \\
& G^{\prime \prime}(f)=\left|G^{*}(f)\right| \sin (\pi \alpha(f) / 2) \\
& \alpha(f)=\left.\frac{\partial \ln \left\langle\Delta r^{2}(t)\right\rangle \mid}{\partial \ln t}\right|_{t=1 / f}
\end{aligned}
$$

where $G^{\prime}$ is storage modulus and $\boldsymbol{G}^{\prime \prime}$ is loss modulus. Figure 6 shows the viscoelastic properties of NaDC hydrogels as a function of frequencies. We can clearly see that, as expected from the temporal evolution of the MSD shown in Figure 5, the viscous component dominates at both low and high frequency, $G^{\prime \prime}(\omega)>$ $G^{\prime}(\omega)$. While the elastic component dominates in the intermediate range, $G^{\prime}(\omega)>G^{\prime \prime}(\omega)$. The viscoelastic behavior is typical to complex systems consisting of worm-like micelles. Some specific aspects of this measurement are worth discussing. First we note that the frequency range is higher than in previous microrheology studies based on diffusive wave spectroscopy (DWS) [37]-[40]. The inherently high sensitivity of the interferometric detection in LC-DLS allows the use of small size probes. In our case, the loaded probes are polystyrene particles with a diameter of $100 \mathrm{~nm}$, which is smaller than that of the tracers traditionally used in DWS-based microrheology [41]. This is rather beneficial since small particles will have only marginal influences on the cavities of the nanofiber network of NaDC hydrogels.

From the frequency-dependent viscoelastic moduli, we can readily obtain two key rheological parameters from the first crossing point where $G^{\prime}(\omega)=G^{\prime \prime}(\omega)$ at $\omega=1 / \tau_{r}$, on the basis of Maxwell's model namely the typical relaxation time and the plateau modulus, $G_{0}=2 G^{\prime}\left(\omega_{r}\right)=2 G^{\prime \prime}\left(\omega_{r}\right)$ [41]. However, the second crossing point in Figure 6 indicates the presence of more complex dynamics. This can be better explained from the Cole-Cole representation of the complex viscoelastic modulus (Figure 6). In this representation, a purely Maxwellian fluid would be described as a semicircle. However, it can be clearly seen from Figure 6 that the hydrogel system deviates from the semicircular behavior predicted by the single exponential stress relaxa-

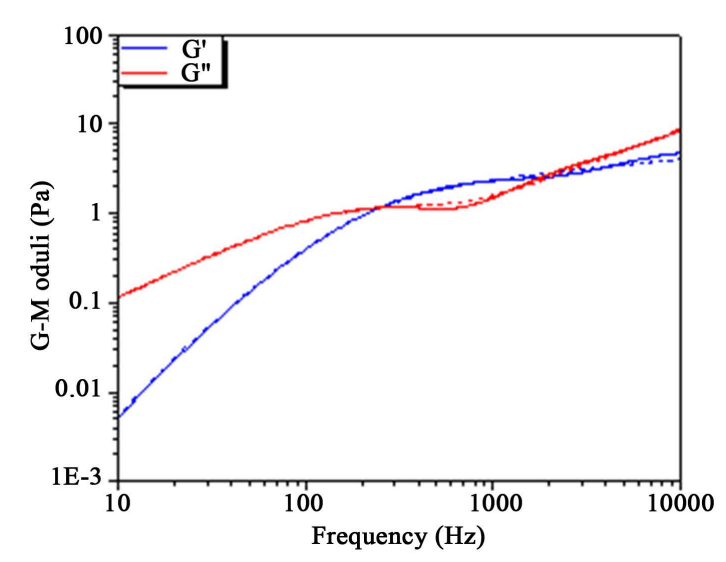

(a)

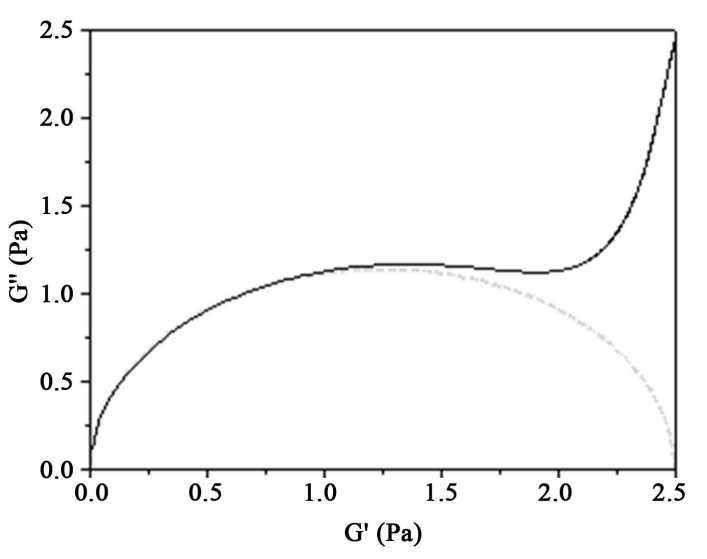

(b)

Figure 6. (a) Frequency-dependent $G^{\prime}$ and $G^{\prime \prime}$ of NaDC hydrogels calculated from LC-DLS technique; (b) ColeCole representation, in which $G^{\prime}$ and $G^{\prime \prime}$ are plotted against each other. The deviation from the semicircle associated to single-relaxation Maxwellian fluids (dashed line) indicates the presence of more complex dynamics. 
tion decay of Maxwellian fluids [42] [43]. This is expected from the various stress relaxation mechanisms observed in the power spectrum as it is accurately described by the superposition of multiple Lorentzian functions. The deviation from the Maxwellian behavior is the fingerprint of Rouse-like dynamics related to reptation and breaking of worm-like micelles [44] [45]. For the gel systems consisting of particle probes loaded into a slightly interconnected worm-like micelle network with weak cross-linkings, the first and second crossing points, i.e. slow and fast dynamics, indicate that breaking events occur faster than reputation [46].

\section{Conclusion}

In conclusion, we form a hydrogel by the self-assembly of NaDC in aqueous solution with sodium chloride at pH-7.0. The NaDC hydrogel with nanofiber networks shows pH-dependent swelling behaviors. By using the polystyrene particles loaded in the hydrogel as a light scattering probe, we study the microrheology of the hydrogel, which shows complex viscoelastic properties. The viscous component dominates at both low and high frequencies, while the elastic component dominates in the intermediate range. The average cavity size of the nanofiber network is estimated to be $\sim 180 \mathrm{~nm}$. The DOX loaded in the NaDC hydrogel can be slowly released into aqueous solution. The release ratio of the DOX is found to increase with the increase of $\mathrm{pH}$ values. By analyzing the release profile of the DOX with Ritger-Peppas equation, we find that the release of the DOX is mainly controlled by the relaxation process of the hydrogels.

\section{Acknowledgements}

This work was supported by the National Science Foundation (CBET 0855322).

\section{References}

[1] Dastidar, P. (2008) Supramolecular Gelling Agents: Can They Be Designed? Chemical Society Reviews, 37, $2699-2715$. http://dx.doi.org/10.1039/b807346e

[2] van Esch, J.H. (2009) We Can Design Molecular Gelators, But Do We Understand Them? Langmuir, 25, $8392-8394$. http://dx.doi.org/10.1021/la901720a

[3] Svobodová, H., Noponen, V., Kolehmainen, K. and Sievänen, E. (2012) Recent Advances in Steroidal Supramolecular Gels. RSC Advances, 2, 4985-5007. http://dx.doi.org/10.1039/c2ra01343f

[4] Buerklea, L.E. and Rowan, S.J. (2012) Supramolecular Gels Formed From Multi-Component Low Molecular Weight Species. Chemical Society Reviews, 41, 6089-6102. http://dx.doi.org/10.1039/c2cs35106d

[5] Babu, S.S., Praveen, V.K. and Ajayaghosh, A. (2014) Functional П-Gelators and Their Applications. Chemical Reviews, 114, 1973-2129. http://dx.doi.org/10.1021/cr400195e

[6] Hofmann, A.F. and Small, D.M. (1967) Detergent Properties of Bile Salts: Correlation with Physiological Function. Annual Review of Medicine, 18, 333-376. http://dx.doi.org/10.1146/annurev.me.18.020167.002001

[7] Mukhopadhyay, S. and Maitra, U. (2004) Chemistry and Biology of Bile Acids. Current Science, 87, 1666-1683.

[8] Terech, P. and Talmon, Y. (2002) Aqueous Suspensions of Steroid Nanotubules: Structural and Rheological Characterizations. Langmuir, 18, 7240-7244. http://dx.doi.org/10.1021/la025574r

[9] Terech, P., Sangeetha, N.M., Demé, B. and Maitra, U. (2005) Self-Assembled Networks of Ribbons in Molecular Hydrogels of Cationic Deoxycholic Acid Analogues. Journal Physics Chemistry B, 109, 12270-12276. http://dx.doi.org/10.1021/jp050666l

[10] Qiao, Y., Lin, Y., Yang, Z., Chen, H., Zhang, S., Yan, Y. and Huang, J. (2010) Unique Temperature-Dependent Supramolecular Self-Assembly: From Hierarchical 1D Nanostructures to Super Hydrogel. Journal Physics Chemistry B, 114, 11725-11730. http://dx.doi.org/10.1021/jp1047369

[11] Mangisi, N., Leggio, C., Jover, A.J., Meijide, F., Pavel, N.V., Tellini, V.H.S., Tato, J.V., Agostino, R.G. and Galantini, L. (2010) Catanionic Tubules with Tunable Charge. Angewandte Chemie International Edition, 49, 6604-6607. http://dx.doi.org/10.1002/anie.201000951

[12] Pal, A., Basit, H., Sen, S., Aswal, V.K. and Bhattacharya, S.J. (2009) Structure and Properties of Two Component Hydrogels Comprising Lithocholic Acid and Organic Amines. Journal of Materials Chemistry, 19, 4325-4334. http://dx.doi.org/10.1039/b903407b

[13] Zhang, X., Zou, J., Tamhane, K., Kobzeff, F. and Fang, J.Y. (2010) Self-Assembly of pH-Switchable Spiral Tubes: Supramolecular Chemical Springs. Small, 6, 217-220. http://dx.doi.org/10.1002/smll.200901067 
[14] Tamhane, K., Zhang, X., Zou, J. and Fang, J.Y. (2010) Assembly and Disassembly of Tubular Spherulites. Soft Matter, 6, 1224-1228. http://dx.doi.org/10.1039/b915183d

[15] Meijide, F., Antelo, A., Alcalde, M.A., Jover, A., Galantini, L., Pavel, N.V. and Tato, J.V. (2010) Supramolecular Structures Generated by Ap-Tert-Butylphenylamide Derivative of Deoxycholic Acid. From Planar Sheets to Tubular Structures through Helical Ribbons. Langmuir, 26, 7768-7773. http://dx.doi.org/10.1021/la904548k

[16] Liu, C., Cui, J., Song, A. and Hao, J. (2011) A Bile Acid-Induced Aggregation Transition and Rheological Properties in Its Mixtures with Alkyltrimethylammonium Hydroxide. Soft Matter, 7, 8952-8960. http://dx.doi.org/10.1039/c1sm05635b

[17] Zhang, X., Bera, T., Liang, W. and Fang J.Y. (2011) Longitudinal Zipping/Unzipping of Self-Assembled Organic Tubes. Journal Physics Chemistry B, 115, 14445-14459. http://dx.doi.org/10.1021/jp2064276

[18] Terech, P., Velu, S.K.P., Pernot, P. and Wiegart, L. (2012) Salt Effects in The Formation of Self-Assembled Lithocholate Helical Ribbons and Tubes. Journal Physics Chemistry B, 116, 11344-11355. http://dx.doi.org/10.1021/jp305365m

[19] Chakrabarty, A., Maitra, U. and Das, A.D. (2012) Metal Cholate Hydrogels: Versatile Supramolecular Systems for Nanoparticle Embedded Soft Hybrid Materials. Journal of Materials Chemistry, 22, 18268-18274. http://dx.doi.org/10.1039/c2jm34016j

[20] di Gregorio, M.C., .Pavel, M.C., Miragaya, J., Jover, A., Meijide, F., Tato, J.V., Tellini, V.H.S. and Galantini, L. (2013) Catanionic Gels Based on Cholic Acid Derivatives. Langmuir, 29, 12342-12351. http://dx.doi.org/10.1021/la402602d

[21] Wang, H., Xu, W., Song, S., Feng, L., Song, A. and Hao, J. (2014) Hydrogels Facilitated by Monovalent Cations and Their Use as Efficient Dye Adsorbents. Journal Physics Chemistry B, 118, 4693-4701. http://dx.doi.org/10.1021/jp500113h

[22] Sun, X., Xin, X., Tang, N., Guo, L., Wang, L. and Xu, G. (2014) Manipulation of the Gel Behavior of Biological Surfactant Sodium Deoxycholate by Amino Acids. Journal Physics Chemistry B, 4, 118, 824-832. http://dx.doi.org/10.1021/jp409626s

[23] Rich, A. and Blow, D.B. (1958) Formation of a Helical Steroid Complex. Nature, 182, 423-427. http://dx.doi.org/10.1038/182423a0

[24] Blow, D.B. and Rich, A. (1960) Studies on the Formation of Helical Deoxycholate Complexes1, 2. Journal of American Chemistry Society, 82, 3566-3571. http://dx.doi.org/10.1021/ja01499a023

[25] Jover, A., Meijide, F., Núñez, E.R. and Tato, J.V. (1996) Unusual Pyrene Excimer Formation during Sodium Deoxycholate Gelation. Langmuir, 12, 1789-1793. http://dx.doi.org/10.1021/la9506335

[26] Ritger, P.L. and Peppas, N.A. (1987) A Simple Equation for Description of Solute Release I. Fickian and Non-Fickian Release from Non-Swellable Devices in the Form of Slabs, Spheres, Cylinders or Discs. Journal of Controlled Release, 5, 23-36. http://dx.doi.org/10.1016/0168-3659(87)90034-4

[27] Mason, T.G. and Weitz, D.A. (1995) Optical Measurements of Frequency-Dependent Linear Viscoelastic Moduli of Complex Fluids. Physical Review Letters, 74, 1250-1253. http://dx.doi.org/10.1103/PhysRevLett.74.1250

[28] Gardel, M.L., Valentine, M.T. and Weitz, D.A. (2003) Microrheology. In: Breuer, K., Ed., Microscale Diagnostic Techniques, Springer-Verlag, New York.

[29] Popescu, G. and Dogariu, A. (2001) Dynamic Light Scattering in Localized Coherence Volumes. Optics Letters, 26, 551-553. http://dx.doi.org/10.1364/OL.26.000551

[30] Popescu, G., Dogariu, A. and Rajagopalan, R. (2002) Spatially Resolved Microrheology Using Localized Coherence Volumes. Physical Review E, 65, Article ID: 041504. http://dx.doi.org/10.1103/PhysRevE.65.041504

[31] Sohn, I.S., Rajagopalan, R. and Dogariu, A.C. (2004) Spatially Resolved Microrheology through a Liquid/Liquid Interface. Journal of Colloid. Interface Science, 269, 503-513. http://dx.doi.org/10.1016/S0021-9797(03)00728-8

[32] Berne, B.J. and Pecora, R. (2000) Dynamic Light Scattering: With Applications to Chemistry, Biology, and Physics. Courier Dover Publications, Mineola.

[33] Jvan Zanten, H. and Rufener, K.P. (2000) Brownian Motion in a Single Relaxation Time Maxwell Fluid. Physical Review E, 62, 5389-5396. http://dx.doi.org/10.1103/PhysRevE.62.5389

[34] Bellour, M., Skouri, M., Munch, J.P. and Hébraud, P. (2002) Brownian Motion of Particles Embedded in a Solution of Giant Micelles. European Physical Journal E, 8, 431-436. http://dx.doi.org/10.1140/epje/i2002-10026-0

[35] Galvan-Miyoshi, J. Delgado, J. and Castillo, R. (2008) Diffusing Wave Spectroscopy in Maxwellian Fluids. European Physical Journal E, 26, 369-377. http://dx.doi.org/10.1140/epje/i2007-10335-8

[36] Mason, T.G. (2000) Estimating the Viscoelastic Moduli of Complex Fluids Using the Generalized Stokes-Einstein Equation. Rheologica Acta, 39, 371-378. http://dx.doi.org/10.1007/s003970000094

[37] Mason, T.G., Gang, H. and Weitz, D.A. (1997) Diffusing-Wave-Spectroscopy Measurements of Viscoelasticity of 
Complex Fluids. Journal of the Optical Society of American, 14, 139-149. http://dx.doi.org/10.1364/JOSAA.14.000139

[38] Palmer, A., Mason, T.G., Xu, J., Kuo, S.C. and Wirtz, D. (1999) Diffusing Wave Spectroscopy Microrheology of Actin Filament Networks. Biophysical Journal, 76, 1063-1071. http://dx.doi.org/10.1016/S0006-3495(99)77271-1

[39] Narita, T., Knaebel, A., Munch, J.P. and Candau, S.J. (2001) Microrheology of Poly(vinyl alcohol) Aqueous Solutions and Chemically Cross-Linked Gels. Macromolecules, 34, 8224-8231. http://dx.doi.org/10.1021/ma010890i

[40] Xu, J., Tseng, Y., Carriere, C.J. and Wirtz, D. (2002) Microheterogeneity and Microrheology of Wheat Gliadin Suspensions Studied by Multiple-Particle Tracking. Biomacromolecules, 3, 92-99. http://dx.doi.org/10.1021/bm015586b

[41] Cardinaux, F., Cipelletti, L., Scheffold, F. and Schurtenberger, P. (2002) Microrheology of Giant-Micelle Solutions. Europhysics Letters, 57, 738-744. http://dx.doi.org/10.1209/epl/i2002-00525-0

[42] Shikata, T., Hirata, H. and Kotaka, T. (1987) Micelle Formation of Detergent Molecules in Aqueous Media: Wiscoelastic Properties of Aqueous Cetyltrimethylammonium Bromide Solutions. Langmuir, 3, 1081-1086. http://dx.doi.org/10.1021/la00078a035

[43] Berret, J.F., Appell, A. and Porte, G. (1993) Linear Rheology of Entangled Wormlike Micelles. Langmuir, 9, 28512854. http://dx.doi.org/10.1021/la00035a021

[44] Edwards, S.F. and Doi, M. (1986) The Theory of Polymer Dynamics. Clarendon, Oxford.

[45] Granek, R. and Cates, M.E. (1992) Stress Relaxation in Living Polymers: Results from a Poisson Renewal Model. Journal of Chemical Physics, 96, 4758-4767. http://dx.doi.org/10.1063/1.462787

[46] Sarmiento-Gomez, E., Santamaría-Holek, I. and Castillo, R. (2014) Mean-Square Displacement of Particles in Slightly Interconnected Polymer Networks. Journal Physics Chemistry B, 118, 1146-1158. 